\title{
General bounds for identifying codes in some infinite regular graphs
}

\author{
Irène Charon \\ CNRS \& ENST \\ 46, rue Barrault \\ 75634 Paris Cedex 13 - France \\ charon@infres.enst.fr \\ Olivier Hudry \\ CNRS \& ENST \\ 46, rue Barrault \\ 75634 Paris Cedex 13 - France \\ hudry@infres.enst.fr
}

\section{Iiro Honkala*}

University of Turku

Department of Mathematics

20014 Turku, Finland

honkala@utu.fi

Antoine Lobstein

CNRS \& ENST

46, rue Barrault

75634 Paris Cedex 13 - France

lobstein@infres.enst.fr

Submitted: October 10, 2000; Accepted: November 14, 2001.

MR Subject Classifications: 05C70 (68R10, 94B65)

\begin{abstract}
Consider a connected undirected graph $G=(V, E)$ and a subset of vertices $C$. If for all vertices $v \in V$, the sets $B_{r}(v) \cap C$ are all nonempty and pairwise distinct, where $B_{r}(v)$ denotes the set of all points within distance $r$ from $v$, then we call $C$ an $r$-identifying code. We give general lower and upper bounds on the best possible density of $r$-identifying codes in three infinite regular graphs.
\end{abstract}

\section{Introduction}

Let $G=(V, E)$ be a connected undirected graph, finite or infinite; we define $B_{r}(v)$, the ball of radius $r$ centred at a vertex $v \in V$, by

$$
B_{r}(v)=\{x \in V: d(x, v) \leq r\},
$$

where $d(x, v)$ denotes the number of edges in any shortest path between $v$ and $x$. Whenever $d(x, v) \leq r$, we say that $x$ and $v$-cover each other (or simply cover if there is no ambiguity). A set of vertices covers a vertex if at least one of its elements does.

${ }^{*}$ Research supported by the Academy of Finland, Grant 44002. 
We call any nonempty subset $C$ of $V$ a code and its elements codewords. A code $C$ is called $r$-identifying, or identifying, if the sets $B_{r}(v) \cap C, v \in V$, are all nonempty and pairwise distinct. The set $B_{r}(v) \cap C$ is called the $r$-identifying set, or identifying set, of $v$ and will be denoted by $I(v)$. Two vertices which have different identifying sets are said to be r-separated, or separated.

The concept of identifying code was introduced in [14]. It was further studied, for different types of graphs, e.g., in [1]-[13].

In this paper we will study the following three 2-dimensional infinite grids:

- $G_{H}$, the brick wall (or hexagonal) grid, with vertex set $V=\mathbb{Z} \times \mathbb{Z}$ and edge set

$$
E_{H}=\left\{\{u=(i, j), v\}: u-v \in\left\{\left(0,(-1)^{i+j+1}\right),( \pm 1,0)\right\}\right\} .
$$

- $G_{S}$, the square lattice, with same vertex set and edge set

$$
E_{S}=\{\{u, v\}: u-v \in\{(0, \pm 1),( \pm 1,0)\}\}
$$

- $G_{T}$, the triangular lattice, or square lattice with one diagonal, with same vertex set and edge set

$$
E_{T}=\{\{u, v\}: u-v \in\{(0, \pm 1),( \pm 1,0),(1,1),(-1,-1)\}\}
$$

See Figures 1, 13, 2 and 3. Note that in these three graphs, $C=V$ is $r$-identifying for all $r$.

Denote by $Q_{n}$ the set of vertices $(x, y) \in V=\mathbb{Z} \times \mathbb{Z}$ with $|x| \leq n$ and $|y| \leq n$. Then we define the density of a code $C$ as

$$
D(C)=\limsup _{n \rightarrow \infty} \frac{\left|C \cap Q_{n}\right|}{\left|Q_{n}\right|}
$$

For a given graph $G=(V, E)$ and a given integer $r$, we search for $r$-identifying codes with minimum density, denoted by $D(G, r)$.

The paper is organized as follows: in Section 2, we improve lower bounds on $D(G, r)$ for the triangular and square lattices, as well as for the brick wall grid, valid for all values of $r$. Sections 3 and 4 give general constructions in the triangular lattice and brick wall grid, respectively. In the Conclusion, we gather all the general results known to us for these three grids and show their asymptotical behaviour (when $r$ goes to infinity).

Note that in [4], the results proved here are announced, and the three grids are studied for small values of $r$. The square lattice with two diagonals is considered in [4] and [3].

\section{General lower bounds}

We consider here any of the three aforementioned infinite graphs and denote it by $G=$ $(V, E)$. For $u, v \in V$, we denote by $\Delta_{r}(u, v)$ the symmetric difference between $B_{r}(u)$ and $B_{r}(v)$. The set $C \cap \Delta_{r}(u, v)$ is the set of codewords $r$-separating $u$ from $v$, and is therefore nonempty if $C$ is $r$-identifying. 


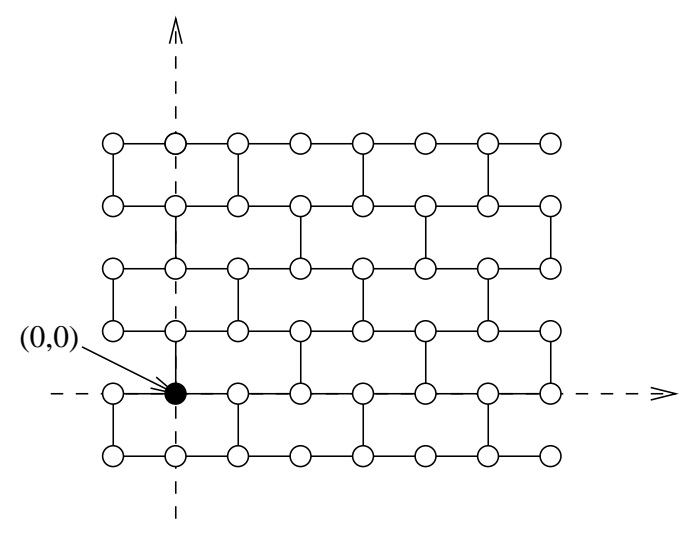

Figure 1: The brick wall (hexagonal) grid (part).

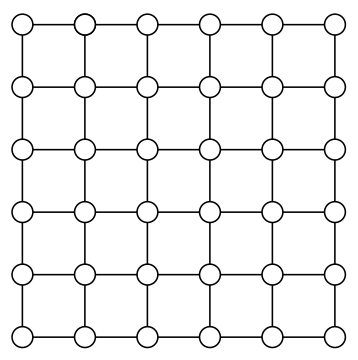

Figure 2: The square lattice (part).

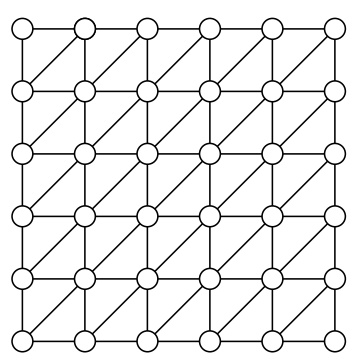

Figure 3: The triangular lattice (part). 
Proposition 1 We consider three vertices $x, y, z \in V$ and an r-identifying code $C \subseteq V$. The set $H_{r}(x, y, z)=\Delta_{r}(x, y) \cup \Delta_{r}(y, z) \cup \Delta_{r}(z, x)$ contains at least two codewords.

Proof. Since $C$ is $r$-identifying, $\Delta_{r}(x, y)$ contains at least one codeword, $c$. We can assume, without loss of generality, that $d(x, c) \leq r$ and $d(y, c)>r$.

First case. Assume that $d(z, c) \leq r$. The vertices $x$ and $z$ are not separated by $c$; so there is another codeword $c^{\prime}$ separating $x$ from $z ; c^{\prime}$ also belongs to $H_{r}(x, y, z)$; the proposition is true.

Second case. Assume now that $d(z, c)>r$. The vertices $y$ and $z$ are not separated by $c$; so there is another codeword $c^{\prime}$ separating them; $c^{\prime}$ also belongs to $H_{r}(x, y, z)$; the proposition is true.

Note that this proposition holds for any connected graph. We define the size of a finite set $S \subset \mathbb{Z}^{2}$ as the maximum between the width of $S$ (which is the difference between the maximum and the minimum abscissae of points of $S$ ) and the height of $S$ (which is the difference between the maximum and the minimum ordinates of points of $S$ ).

Proposition 2 Consider a finite set $E_{0,0} \subseteq \mathbb{Z}^{2}$, with cardinality e. Denote by $E_{i, j}$ the set of points obtained from $E_{0,0}$ by a translation of vector $(i, j)$, and set $\mathcal{E}=\left\{E_{i, j}: i \in \mathbb{Z}, j \in\right.$ $\mathbb{Z}\}$. Then each point in $\mathbb{Z}^{2}$ belongs to e elements $E_{i, j}$ of $\mathcal{E}$.

Proof. Let $E_{0,0}=\left\{a_{1}, \ldots, a_{e}\right\}$. Now $x \in E_{i, j}$ if and only if $x=a_{k}+(i, j)$ for some $k$ in $\{1, \ldots, e\}$, that is, if and only if $(i, j)=x-a_{k}$; so there are exactly $e$ choices for $(i, j)$.

Theorem 1 improves on $D\left(G_{T}, r\right) \geq 1 /(4 r+2)$ from [9].

Theorem 1 The minimum density of an r-identifying code in the triangular lattice satisfies

$$
D\left(G_{T}, r\right) \geq \frac{2}{6 r+3} .
$$

Proof. We call a triangle any 3 -tuple $(x, y, z)$ such that there exist $i \in \mathbb{Z}$ and $j \in \mathbb{Z}$ with $x=(i, j), y=(i, j+1)$ and $z=(i+1, j+1)$, see Figures 4 and 5 .

It is easy to check that, in the triangular lattice:

$$
\mid H_{r}(\text { triangle }) \mid=6 r+3 .
$$

Moreover, $H_{r}((i, j),(i, j+1),(i+1, j+1))$ is the translate of $H_{r}((0,0),(0,1),(1,1))$ by the vector $(i, j)$. Now we can use Proposition 2 with $E_{0,0}=H_{r}((0,0),(0,1),(1,1))$ : for each vertex $v$ of the lattice, there exist exactly $6 r+3$ triangle $(\mathrm{s})$ such that $H_{r}($ triangle) contains $v$.

Denote by $p$ the size of $H_{r}$ (triangle); we suppose that $C$ is an $r$-identifying code and we consider, with $n \in \mathbb{N}, 2 n \geq p$, the set:

$$
\left\{(\text { triangle }, c): H_{r}(\text { triangle }) \subseteq Q_{n}, c \in C \cap Q_{n}, c \in H_{r}(\text { triangle })\right\} .
$$




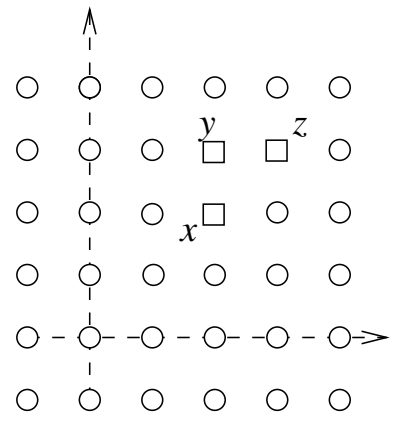

Figure 4: A triangle.
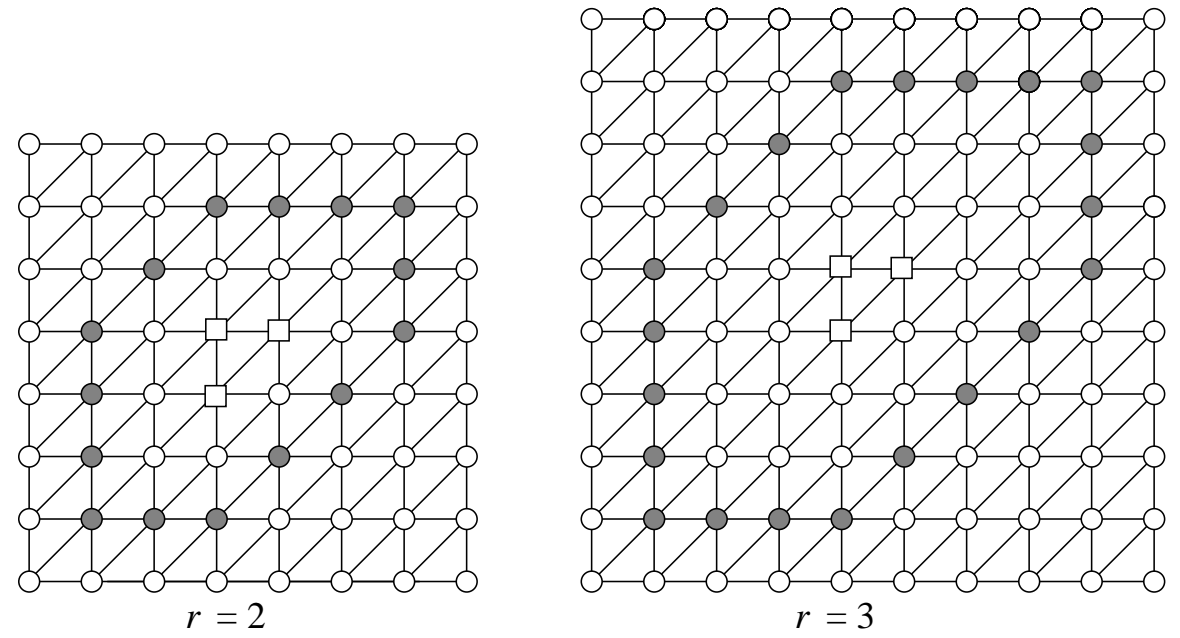

Figure 5: $H_{r}($ triangle) for the triangular lattice. 
Using Proposition 1, we see that the cardinality of this set is at least $2 \times \mid$ \{triangle : $H_{r}($ triangle $\left.) \subseteq Q_{n}\right\} \mid$. On the other hand, it is at most $\left|C \cap Q_{n}\right| \times(6 r+3)$.

Since

$$
\mid\left\{\text { triangle }: H_{r}(\text { triangle }) \subseteq Q_{n}\right\} \mid \geq(2 n-p+1)^{2},
$$

we obtain:

$$
\frac{\left|C \cap Q_{n}\right|}{\left|Q_{n}\right|} \geq \frac{2}{6 r+3} \times \frac{(2 n-p+1)^{2}}{(2 n+1)^{2}} .
$$

By letting $n$ tend to infinity, we obtain the result.

Theorem 2 improves on $D\left(G_{H}, r\right) \geq 1 /(4 r+4)$ from [9].

Theorem 2 The minimum density of an r-identifying code in the brick wall grid satisfies $D\left(G_{H}, r\right) \geq \frac{2}{5 r+3}$ if $r$ is even and $D\left(G_{H}, r\right) \geq \frac{2}{5 r+2}$ if $r$ is odd.

Proof. Since the brick wall grid is not globally invariant by all translations, we have to adapt slightly our previous method. We now call a triangle any 3 -tuple $(x, y, z)$ such that there exist $i \in \mathbb{Z}$ and $j \in \mathbb{Z}$ with $x=(i, j), y=(i+1, j), z=(i+2, j)$, see Figure 6 . We denote by $E_{0,0}$ the set $H_{r}((0,0),(1,0),(2,0))$ and $E_{1,0}$ the set $H_{r}((1,0),(2,0),(3,0))$. One can remark that $E_{1,0}$ is obtained from $E_{0,0}$ by the translation of vector $(1,0)$ followed by the symmetry with respect to the X-axis. Denote by $E_{i, j}$ the set of vertices obtained:

- if $i+j$ is even, from $E_{0,0}$ by the translation of vector $(i, j)$;

- if $i+j$ is odd, from $E_{1,0}$ by the translation of vector $(i-1, j)$.

It is clear that $E_{i, j}$ is the set $H_{r}((i, j),(i+1, j),(i+2, j))$.

We consider also the set $\mathcal{E}=\left\{E_{i, j}: i \in \mathbb{Z}, j \in \mathbb{Z}\right\}$. One can observe, using considerations of horizontal symmetry, first that the sets $E_{i, j}$ all have the same cardinality, denoted here by $e$, and second that the number of times a vertex is in a set $E_{i, j}$ does not depend on the considered vertex; it follows, using the same type of argument as in the proof of Proposition 2, that each vertex of the infinite graph belongs to $e$ elements $E_{i, j} \in \mathcal{E}$.

Now, the proof is nearly the same as the previous proof; it is only necessary to compute the value of $e$ to obtain the result.

One readily checks that, for the brick wall grid:

$-\mid H_{r}($ triangle $) \mid=5 r+3$ if $r$ is even;

$-\mid H_{r}($ triangle $) \mid=5 r+2$ if $r$ is odd.

Remark. With the same argument, it is possible to show that the minimum density of an $r$-identifying code in the square lattice satisfies

$$
D\left(G_{S}, r\right) \geq \frac{2}{6 r+3}
$$

but the following theorem will give a better lower bound, also improving on $D\left(G_{S}, r\right) \geq$ $2 /(7 r+4)$ from [13]. 

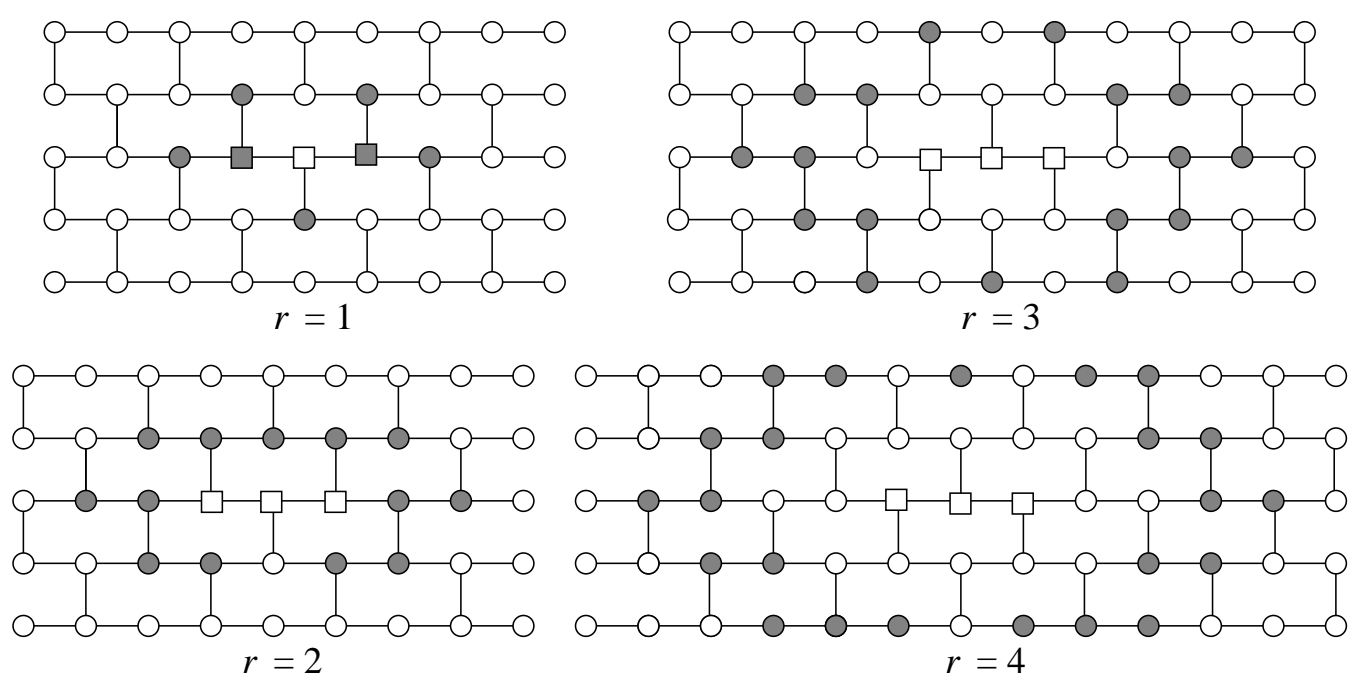

Figure 6: $H_{r}($ triangle) for the brick wall grid.

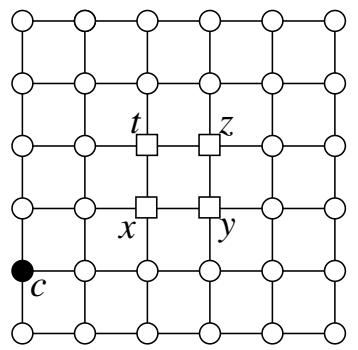

Figure 7: A square.

Theorem 3 The minimum density of an r-identifying code in the square lattice satisfies

$$
D\left(G_{S}, r\right) \geq \frac{3}{8 r+4} .
$$

Proof. In this proof, we call a square any 4 -tuple $(x, y, z, t)$ such that there exist $i \in \mathbb{Z}$ and $j \in \mathbb{Z}$ with $x=(i, j), y=(i+1, j), z=(i+1, j+1)$ and $t=(i, j+1)$, see Figure 7 . Consider the set

$$
K_{r}(x, y, z, t)=\Delta_{r}(x, y) \cup \Delta_{r}(x, z) \cup \Delta_{r}(x, t) \cup \Delta_{r}(y, z) \cup \Delta_{r}(y, t) \cup \Delta_{r}(z, t),
$$

see Figure 8.

We will prove first that, if we have an $r$-identifying code for the square lattice, then $K_{r}(x, y, z, t)$ contains at least three codewords (cf. Proposition 1). We can assume, without loss of generality, that $K_{r}(x, y, z, t)$ contains a codeword $c=\left(i^{\prime}, j^{\prime}\right)$ with: $i^{\prime} \leq i$ and $j^{\prime} \leq j$. We have:

$$
d(c, y)=d(c, x)+1, d(c, z)=d(c, x)+2, d(c, t)=d(c, x)+1,
$$



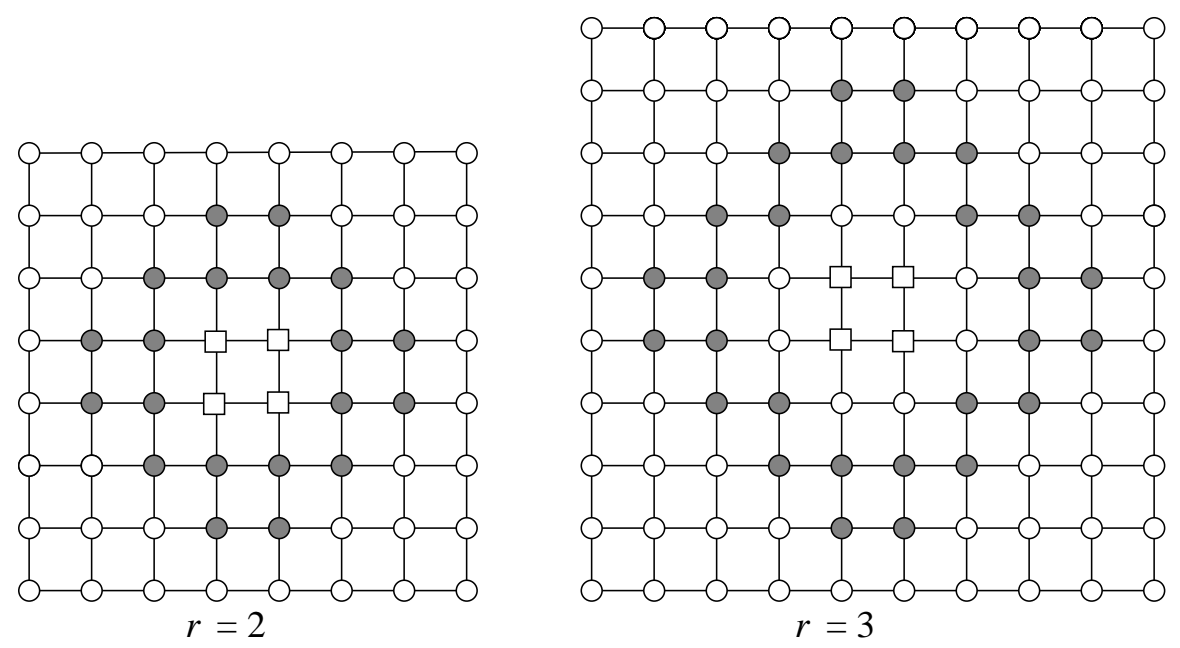

Figure 8: $K_{r}($ square $)$ for the square lattice.

cf. Figure 7. Since $x$ belongs to $K_{r}(x, y, z, t), d(c, x)=r-1$ or $d(c, x)=r$. First, if $d(c, x)=r$, then by (1), $c$ does not cover $y, z$ or $t$, and thus does not belong to $\Delta_{r}(y, z) \cup \Delta_{r}(z, t) \cup \Delta_{r}(y, t)$, which is $H_{r}(y, z, t)$; from Proposition $1, H_{r}(y, z, t)$ contains at least two codewords and they are distinct from $c$; so $K_{r}(x, y, z, t)$ contains at least three codewords.

If $d(c, x)=r-1$, then $c$ does not separate between $x, y$ and $t$, i.e., $c$ does not belong to $H_{r}(x, y, t)$. Using again Proposition 1 , we see that $K_{r}(x, y, z, t)$ must contain at least three codewords.

In other words, in the square lattice, one codeword necessarily separates a square into a singleton and a triangle, and the triangle needs two more codewords.

Moreover, it is easy to check that $\left|K_{r}(x, y, z, t)\right|=8 r+4$. Using the same argument as in the proof of Theorem 1, replacing triangle by square, and the two codewords for a triangle by three codewords for a square, we obtain the result.

\section{A general construction for the triangular lattice}

In this section, we denote a vertex $P$ by $P=(i, j)$, and a vertex $P_{k}$ by $P_{k}=\left(i_{k}, j_{k}\right)$. For $i \in \mathbb{Z}$, we set $\varepsilon(i)=0$ if $i$ is even and $\varepsilon(i)=1$ if $i$ is odd.

The distance $d$ between two vertices of the triangular lattice is given by:

- $d\left(P_{1}, P_{2}\right)=\max \left(\left|i_{2}-i_{1}\right|,\left|j_{2}-j_{1}\right|\right)$ if $\left(i_{2}-i_{1}\right) \times\left(j_{2}-j_{1}\right) \geq 0$,

- $d\left(P_{1}, P_{2}\right)=\left|i_{2}-i_{1}\right|+\left|j_{2}-j_{1}\right|$ otherwise.

We give three theorems, corresponding to the cases $r$ odd, $r$ a multiple of 4 and $r$ even and not a multiple of 4 .

Theorem 4 Let $r$ be a positive odd integer. There is an r-identifying code in the triangular lattice with density $\frac{1}{2 r+2}$. 
Proof. Let $r$ be a positive odd integer. We define, for $k$ belonging to $\mathbb{Z}$, a set $C_{k}$ of vertices by:

$$
C_{k}=\{(k(r+1), \alpha): \alpha \in \mathbb{Z}, \alpha \text { even }\} .
$$

We claim that $C$, the union of the sets $C_{k}$ for $k \in \mathbb{Z}$, is an $r$-identifying code for the triangular lattice. Figure 9 illustrates the case $r=5$.

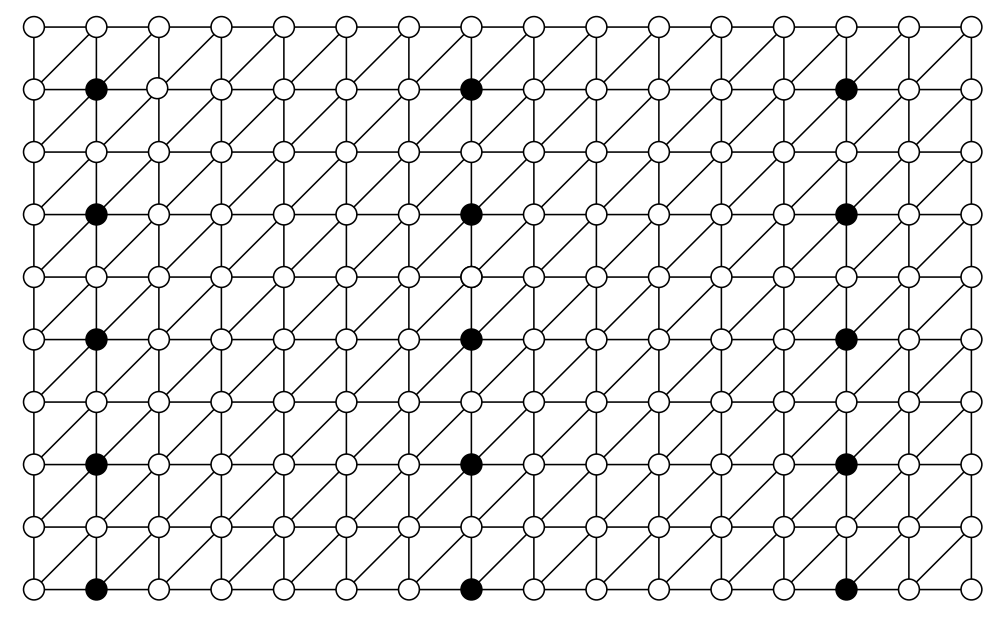

Figure 9: A 5-identifying code for the triangular lattice. Codewords are in black.

A vertex $P=(i, j)$ is $r$-covered by $C_{k}$ if and only if:

$$
k(r+1)-r \leq i \leq k(r+1)+r .
$$

So, all the vertices are covered. Furthermore, the minimum value of $k$, denoted by $k(P)$, $k \in \mathbb{Z}$, such that $P=(i, j)$ is covered by an element of $C_{k}$ is:

$$
k(P)=\left\lceil\frac{i-r}{r+1}\right\rceil .
$$

Now we show that any two vertices $P_{1}$ and $P_{2}$ are $r$-separated.

A vertex $P=(i, j)$ is such that $k(P)=0$ if and only if: $0 \leq i \leq r$. We consider such a vertex and denote by $J_{k}(P)$ the set of ordinates of codewords covering $P$ and belonging to $C_{k}$.

A codeword $(0, \alpha)$ where $\alpha \in \mathbb{Z}$ is even, that is to say a codeword of $C_{0}$, covers $P$ if and only if:

$$
\left\{\begin{array}{l}
i+\alpha-j \leq r \\
j-\alpha \leq r
\end{array}\right.
$$

So:

$$
J_{0}(P)=\{\alpha \in \mathbb{Z}: \alpha \text { even, } j-r+1-\varepsilon(j) \leq \alpha \leq j-i+r-1+\varepsilon(i+j)\} .
$$


In the same way, we obtain that:

if $i=0, J_{1}(P)$ is empty

and otherwise:

$$
J_{1}(P)=\{\alpha \in \mathbb{Z}: \alpha \text { even, } j-i+2-\varepsilon(i+j) \leq \alpha \leq j+r-1+\varepsilon(j)\} .
$$

We consider two distinct vertices $P_{1}$ and $P_{2}$ and we suppose that they are not $r$-separated. This clearly implies that $k\left(P_{1}\right)=k\left(P_{2}\right)$. We can assume, without loss of generality, that $k\left(P_{1}\right)=k\left(P_{2}\right)=0$ (the other cases are obtained by translation). So:

$$
\left\{\begin{array}{l}
0 \leq i_{1} \leq r \\
0 \leq i_{2} \leq r
\end{array}\right.
$$

Suppose first that $i_{1}=i_{2}=0$; by (2), we have:

$$
\left\{\begin{array}{l}
j_{1}-\varepsilon\left(j_{1}\right)=j_{2}-\varepsilon\left(j_{2}\right) \\
j_{1}+\varepsilon\left(j_{1}\right)=j_{2}+\varepsilon\left(j_{2}\right),
\end{array}\right.
$$

so: $j_{1}=j_{2} ; P_{1}=P_{2}$, a contradiction.

Assume now that $i_{1}=0$ and $i_{2}>0 ; J_{1}\left(P_{1}\right)$ is empty and $J_{1}\left(P_{2}\right)$ is not, a contradiction with the fact that $P_{1}$ and $P_{2}$ are not separated.

Suppose finally that $i_{1}>0$ and $i_{2}>0$; we have, by (2) and (3):

$$
\left\{\begin{array}{l}
j_{1}-\varepsilon\left(j_{1}\right)=j_{2}-\varepsilon\left(j_{2}\right) \\
j_{1}-i_{1}+\varepsilon\left(i_{1}+j_{1}\right)=j_{2}-i_{2}+\varepsilon\left(i_{2}+j_{2}\right) \\
j_{1}-i_{1}-\varepsilon\left(i_{1}+j_{1}\right)=j_{2}-i_{2}-\varepsilon\left(i_{2}+j_{2}\right) \\
j_{1}+\varepsilon\left(j_{1}\right)=j_{2}+\varepsilon\left(j_{2}\right) .
\end{array}\right.
$$

We easily deduce that $P_{1}=P_{2}$, again a contradiction.

Theorem 5 If $r \geq 4$ is divisible by four, then there is an $r$-identifying code with density $\frac{1}{2 r+4}$ in the triangular lattice.

Proof. It is now convenient to adopt a new representation of the triangular lattice and draw it as in Figure 10. We denote by $X_{i}, i \in \mathbb{Z}$, and by $Y_{j}, j \in \mathbb{Z}$, the vertical and horizontal lines, respectively, formed by the lattice points. We take as codewords of $C$ all the lattice points in the sets $X_{i} \cap Y_{j}$ with $i$ even, $j$ divisible by $r+2$ and $i / 2 \equiv j /(r+2) \bmod 2$. The case $r=4$ is given in Figure 10. Clearly the density of $C$ is $1 /(2 r+4)$.

Assume that $x$ is an unknown vertex, and that we know $I(x)$. We now show that based on $I(x)$ we can unambiguously identify $x$.

If $j$ is divisible by $r+2$, we see from Figure 11 that $I(x)$ contains at least $r / 2$ codewords of $Y_{j}$ if and only if $x \in Y_{k}$ for some $k$ satisfying the condition $j-r-1 \leq k \leq j+r+1$ or $x$ is a codeword in $Y_{j-r-2}$ or $Y_{j+r+2}$. In particular, $x$ is a codeword if and only if there is an index $j$ such that $I(x)$ contains $r / 2$ codewords from $Y_{j}$ and $r / 2$ from $Y_{j-2 r-4}$. And 


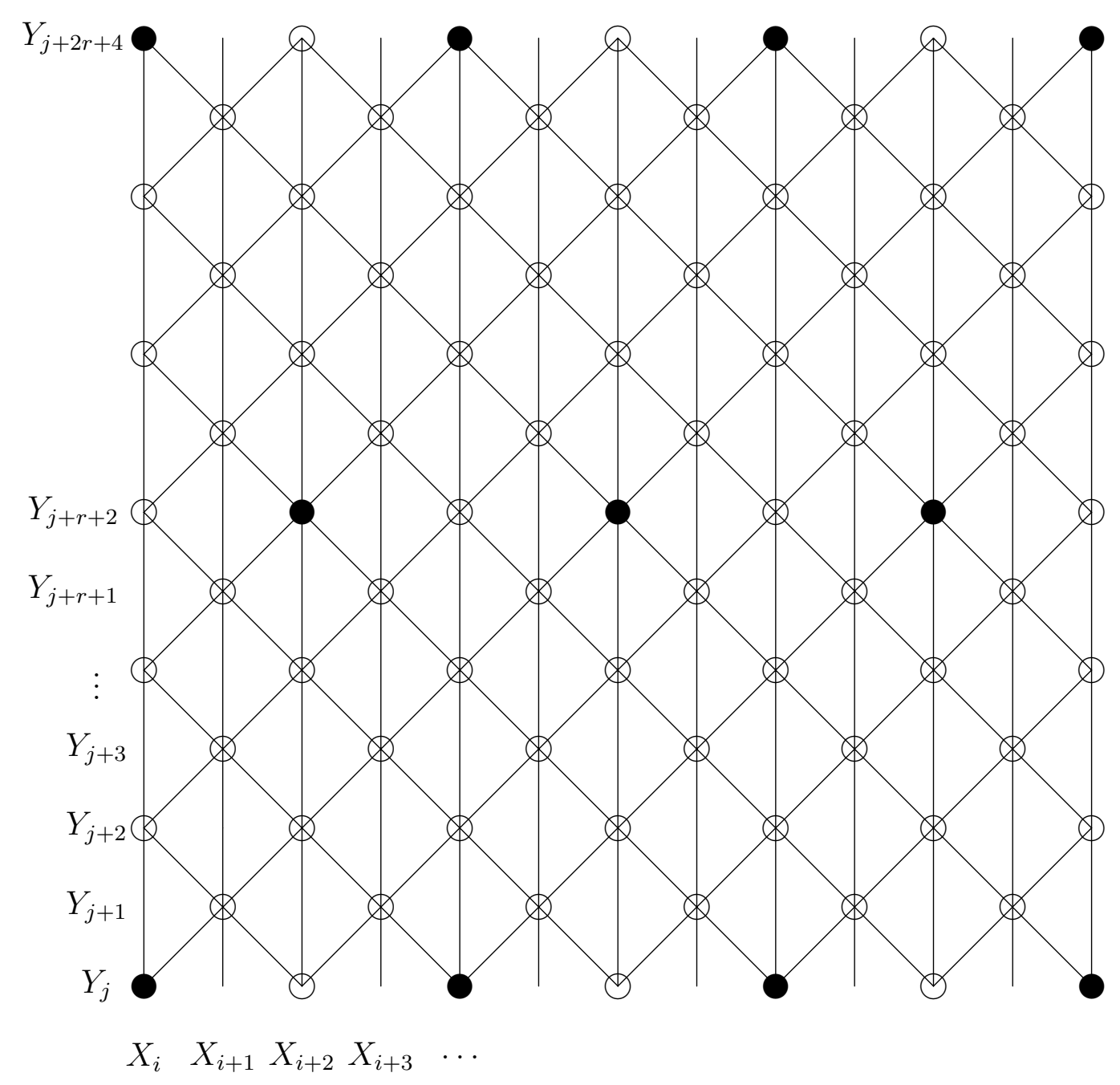

Figure 10: An $r$-identifying code with density $1 /(2 r+4)$ for the triangular lattice when $r=4$. Codewords are in black. 


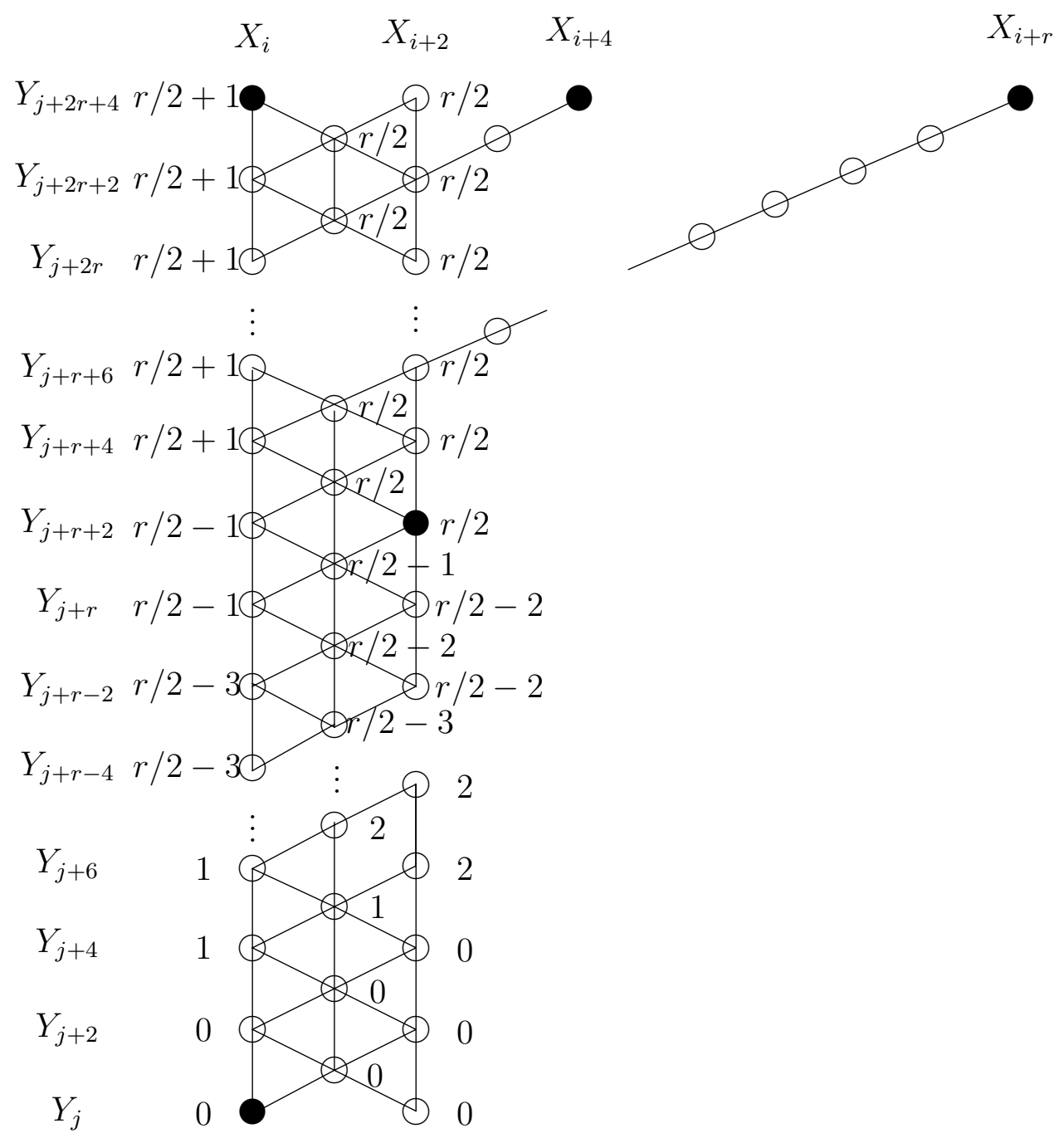

Figure 11: The number beside a vertex $x$ indicates how many codewords in $Y_{j+2 r+4}$ $r$-cover $x$. 
if so, then $x \in Y_{j-r-2}$ and we know that it is the middle point of $I(x) \cap Y_{j-r-2}$. Assume therefore that $x$ is not a codeword.

Let $j$ be the smallest index such that $\left|I(x) \cap Y_{j}\right| \geq r / 2$. Then $x$ is either a noncodeword in $Y_{j}$ or is in some $Y_{k}, j+1 \leq k \leq j+r+1$.

Let $j^{\prime}$ be the largest index such that $\left|I(x) \cap Y_{j^{\prime}}\right| \geq r / 2$. There are two possibilities: either $j^{\prime}=j$ or $j^{\prime}=j+r+2$. If $j^{\prime}=j$, then we know that $x$ is a noncodeword in $Y_{j}$, and it is the unique point lying between the two middle points of $I(x) \cap Y_{j}$. We can therefore assume that $j^{\prime}=j+r+2$, in which case we know that $x$ is in some $Y_{k}, j+1 \leq k \leq j+r+1$.

Let $Y=\cup_{j+1 \leq k \leq j+r+1} Y_{k}$. If $c \in C \cap Y_{j+r+2} \cap X_{i}$, then $B_{r}(c) \cap Y=Y \cap\left(\cup_{i-r \leq k \leq i+r} X_{k}\right)$. We know that for exactly one of the two lines $Y_{j}$ and $Y_{j+r+2}$, the codewords are in the lines $X_{i}$ with $i$ divisible by four. By taking the left-most codeword on that line that still covers $x$, we find an index $4 t$ such that $x \in X_{4 t-3} \cup X_{4 t-2} \cup X_{4 t-1} \cup X_{4 t}$. Using the other line, and taking the left-most codeword on it that still covers $x$, we find an index $4 t^{\prime}+2$ such that $x \in X_{4 t^{\prime}-1} \cup X_{4 t^{\prime}} \cup X_{4 t^{\prime}+1} \cup X_{4 t^{\prime}+2}$. The intersection of these two gives us an index $2 s$ such that $x \in X_{2 s-1} \cup X_{2 s}$. In the same way, by considering the right-most codewords in $Y_{j+r+2}$ and $Y_{j}$ that still cover $x$, we find an index $2 s^{\prime}$ such that $x \in X_{2 s^{\prime}} \cup X_{2 s^{\prime}+1}$. The intersection of these two finally gives us the index $i$ for which $x \in X_{i}$.

Denote $f(x)=\left|I(x) \cap Y_{j+2 r+4}\right|$ and $g(x)=\left|I(x) \cap Y_{j-r-2}\right|$. Assume that $x \in Y_{j+k}$, $1 \leq k \leq r+1$.

Assume first that there are no codewords on the line $X_{i}$ containing $x$. Then using Figure 11, we see that for $k=1,3,5, \ldots, r+1$ the pairs $(f(x), g(x))$ are $(0, r / 2-1)$, $(0, r / 2-2),(1, r / 2-3),(2, r / 2-4), \ldots,(r / 2-4,2),(r / 2-3,1),(r / 2-2,0),(r / 2-1,0)$ and all these pairs are different (since $r \geq 4$ ), and we can identify $x$.

Assume second that there are codewords on the line $X_{i}$ containing $x$. Without loss of generality the point in $X_{i} \cap Y_{j}$ is a codeword; the case in which $X_{i} \cap Y_{j+r+2}$ is a codeword is symmetric. For $k=2,4, \ldots, r$ the pairs $(f(x), g(x))$ are $(0, r / 2-2),(1, r / 2-2)$, $(1, r / 2-4),(3, r / 2-4), \ldots,(r / 2-3,2),(r / 2-3,0),(r / 2-1,0)$, and we can again identify $x$.

In view of the previous proof it is not suprising that we can modify the construction slightly and obtain the following result.

Theorem 6 If $r$ is even and not divisible by four, then there is an r-identifying code with density $\frac{1}{2 r+2}$ in the triangular lattice.

Proof. We take as codewords of $C$ all the lattice points in the sets $X_{i} \cap Y_{j}$ where $(i, j)$ is of the form $(4 a, 0)+b(2,2 r+2)$ or $(4 a, r+2)+b(2,2 r+2)$ where $a, b \in \mathbb{Z}$. Clearly the density of $C$ is $1 /(2 r+2)$.

Let $x$ be the unknown vertex for which the set $I(x)$ is known.

We follow the same strategy as in the previous proof. As the first step we try to find an index $j$ divisible by $2 r+2$ such that we know that $x \in Y_{k}, j-r-1 \leq k \leq j$ (Case 1) or we know that $x \in Y_{k}, j+1 \leq k \leq j+r$ (Case 2).

From Figure 12 we see that we can choose an index $j^{\prime}$ divisible by $2 r+2$ such that $\left|I(x) \cap Y_{j^{\prime}}\right| \geq r / 2$. Using Figure 12 we see that then either 1) $x \in Y_{k}$ for some $j^{\prime}-r-1 \leq$ 

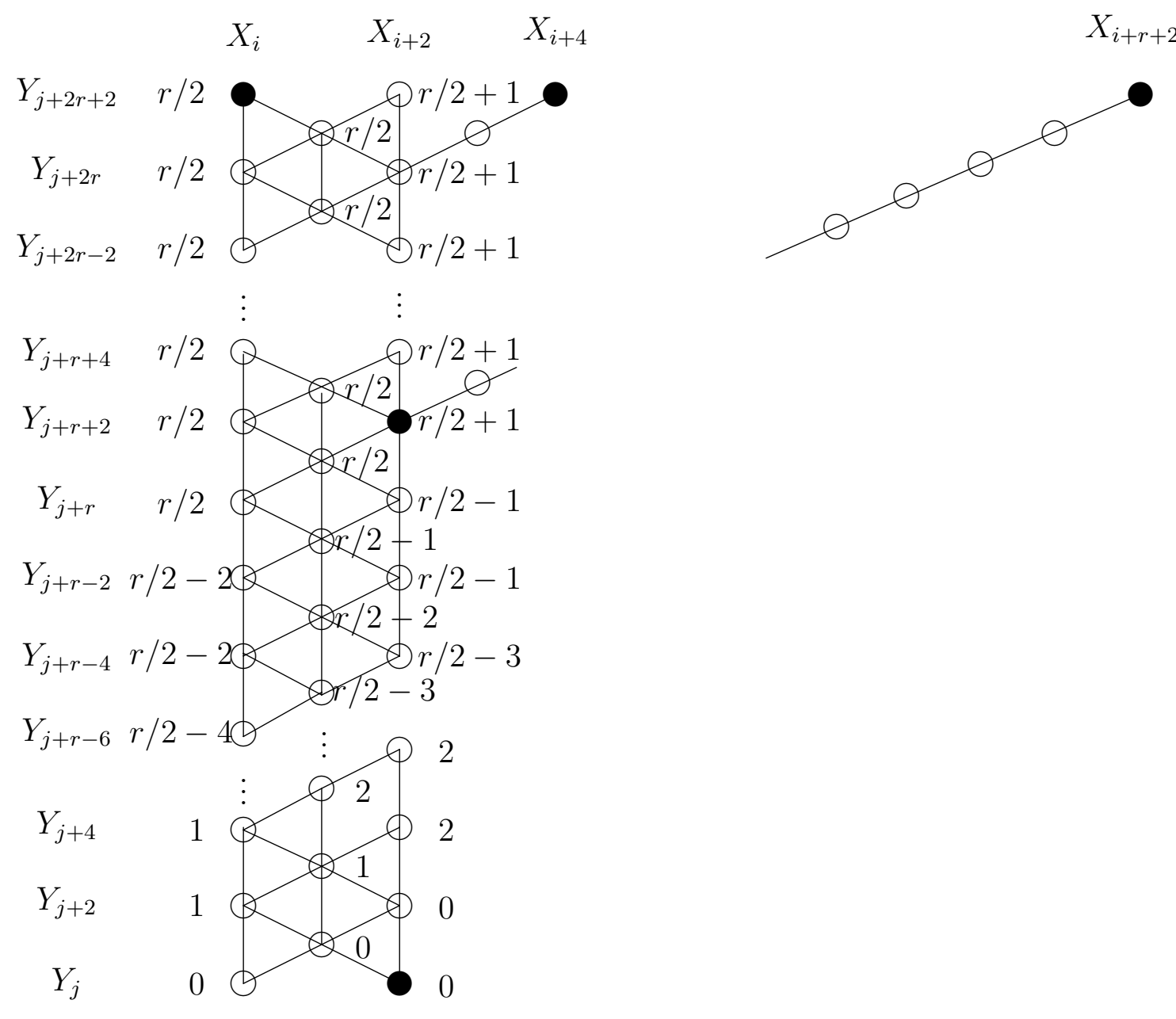

$Y_{j+r+4} \quad r / 2$

$Y_{j+r+2} \quad r / 2$

$Y_{j+r} \quad r / 2$

$Y_{j+r-2} r / 2-2 \bigotimes r / 2-1$

$Y_{j+r-4} r / 2-2 \ngtr r / 2-3$

$Y_{j+r-6} \quad r / 2-4 \varnothing \quad \vdots \bigotimes 2$

$Y_{j+4}$

$Y_{j+2}$

$Y_{j}$

$0 \rightarrow 0$

Figure 12: The number beside a vertex $x$ indicates how many codewords in $Y_{j+2 r+2}$ $r$-cover $x$. 
$k \leq j^{\prime}+r+1$ or 2) $x \in Y_{j^{\prime}-r-2} \cup Y_{j^{\prime}+r+2}$. However, we can immediately check if 2) holds: $\left|I(x) \cap Y_{j^{\prime}+2 r+2}\right|=r / 2+1$ if and only if 2) holds and $x \in Y_{j^{\prime}+r+2}$ : we can then choose $j=j^{\prime}+2 r+2$ and move to Case 1 . Similarly $\left|I(x) \cap Y_{j^{\prime}-2 r-2}\right|=r / 2+1$ if and only if 2) holds and $x \in Y_{j^{\prime}-r-2}$ : we can now choose $j=j^{\prime}-2 r-2$ and move to Case 2 .

So, assume that we have concluded that 2) did not hold. Then we know that $j^{\prime}-r-1 \leq$ $k \leq j^{\prime}+r+1$.

Furthermore, $\left|I(x) \cap Y_{j^{\prime}+r+2}\right| \geq r / 2$ if and only if either $x \in Y_{k}$ for some $j^{\prime}+1 \leq$ $k \leq j^{\prime}+r+1$ or $x$ is a codeword on $Y_{j^{\prime}}$. However, the latter holds if and only if $\left|I(x) \cap Y_{j^{\prime}-r}\right|=r / 2+1$ : if it does, we take $j=j^{\prime}$ and move to Case 1. Finally, $x \in Y_{j^{\prime}+r+1}$ if and only if $\left|I(x) \cap Y_{j^{\prime}+2 r+2}\right|=r / 2$ and $\left|I(x) \cap Y_{j^{\prime}}\right|=r / 2$ : if yes, then we take $j=j^{\prime}+2 r+2$ and move to Case 1. Otherwise, we have been able to decide that either $j^{\prime}-r-1 \leq k \leq j^{\prime}$, then take $j=j^{\prime}$ and move to Case 1 ; or that $j^{\prime}+1 \leq k \leq j^{\prime}+r$, then again take $j=j^{\prime}$ and move to Case 2 .

Case 1: $j-r-1 \leq k \leq j$. Then the codewords on $Y_{j}$ and $Y_{j-r}$ are not above each other, and exactly the same argument as in the previous proof gives us the unique index $i$ for which $x \in X_{i}$. Define $f(x)=\left|I(x) \cap Y_{j+r+2}\right|$ and $g(x)=\left|I(x) \cap Y_{j-2 r-2}\right|$. Assume that $x \in Y_{j-h}, h \in\{0,1,2, \ldots, r+1\}$. If $X_{i} \cap C=\emptyset$, then we can immediately tell on which $Y_{k}$ the vertex $x$ lies, because the values $g(x)$ for $h=1,3, \ldots, r+1$ are all different: $g(x)$ takes all the values from 0 to $r / 2$. Assume then that $X_{i} \cap Y_{j} \subseteq C$. (The remaining cases are symmetric.) Then the pairs $(f(x), g(x))$ for $h=0,2,4, \ldots, r$ are $(r / 2,0),(r / 2-2,0)$, $(r / 2-2,2),(r / 2-4,2), \ldots,(1, r / 2-3),(1, r / 2-1),(0, r / 2-1)$. They are all different, and we can identify $x$.

Case 2: $j+1 \leq k \leq j+r$. Denote $f(x)=\left|I(x) \cap Y_{j+2 r+2}\right|$ and $g(x)=\left|I(x) \cap Y_{j-r}\right|$.

Now the codewords on $Y_{j}$ and $Y_{j+r+2}$ are above each other - without loss of generality the codewords are in the columns $X_{i}$ with $i$ divisible by four - and we cannot immediately deduce the index $i$ for which $x \in X_{i}$. Anyway, using the right-most codeword in $Y_{j+r+2}$ that still covers $x$ we find an index $4 t$ such that $x \in X_{4 t-2} \cup X_{4 t-1} \cup X_{4 t} \cup X_{4 t+1}$ and using the left-most codeword in $Y_{j}$ that covers $x$ we find an index $4 t^{\prime}$ such that $x \in X_{4 t^{\prime}-1} \cup X_{4 t^{\prime}} \cup$ $X_{4 t^{\prime}+1} \cup X_{4 t^{\prime}+2}$. The intersection of these sets is either $X_{4 t-2}$ or $X_{4 t-1} \cup X_{4 t} \cup X_{4 t+1}$; in the latter case, we can then decide whether or not $x \in X_{4 t}$ : if no, then $f(x)+g(x)=r / 2$, which is odd; if yes, then $f(x)+g(x)$ is the sum of two even numbers and hence even.

All these together imply that if $x \in X_{2 s}$ for some $s$, then we know the index $2 s$; and otherwise we have found an index $4 t$ such that $x \in X_{4 t-1} \cup X_{4 t+1}$.

Denote by $h$ the index $(1 \leq h \leq r)$ such that $x \in Y_{j+h}$.

Assume first that $x \in X_{4 t}$ for some $t$. Then the pairs $(f(x), g(x))$ for $h=2,4, \ldots, r$ are $(0, r / 2-1),(2, r / 2-1),(2, r / 2-3), \ldots,(r / 2-3,2),(r / 2-1,2),(r / 2-1,0)$, and we are done.

Assume second that $x \in X_{4 t-2}$ for some $t$. Then the pairs $(f(x), g(x))$ for $h=$ $2,4, \ldots, r$ are $(1, r / 2),(1, r / 2-2),(3, r / 2-2), \ldots,(r / 2-2,3),(r / 2-2,1)(r / 2,1)$, and we are again done.

Assume finally that $x \in X_{4 t-1} \cup X_{4 t+1}$. Then we immediately find the index $k$ for which $x \in Y_{k}$ because all the relevant values of $f(x)$ are different. It suffices to decide whether $x \in X_{4 t-1}$ or $x \in X_{4 t+1}$. We know that $f(x)+g(x)=r / 2$. Since $r / 2$ is odd, 


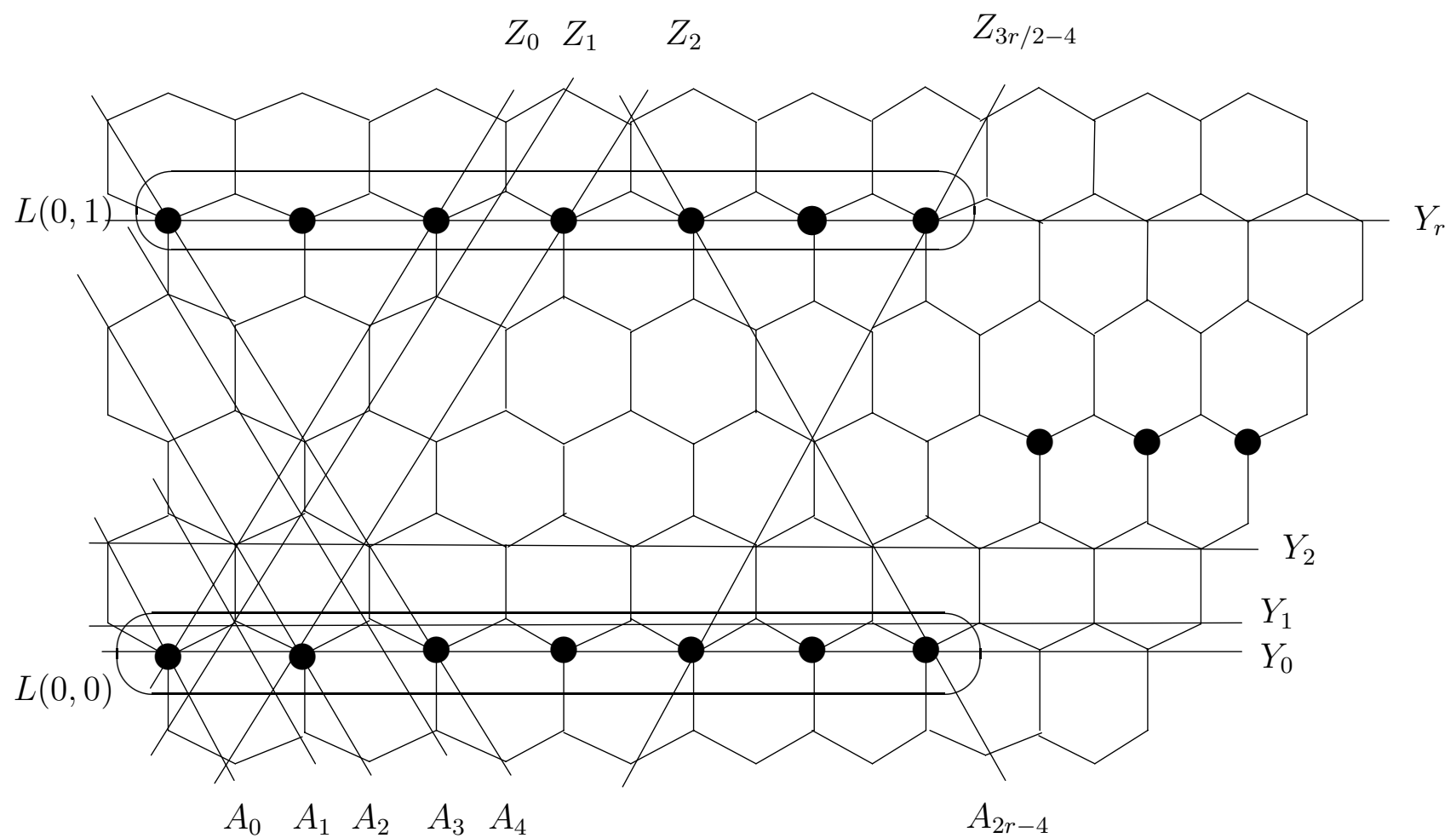

Figure 13: An $r$-identifying code in the hexagonal (brick wall) grid for $r=8$. Codewords are in black.

either $f(x)$ or $g(x)$ is odd; say $f(x)$. Because $X_{4 t} \cap Y_{j+2 r+2}=\emptyset$ (also $X_{4 t} \cap Y_{j-r}=\emptyset$, so the case $g(x)$ odd is similar), we know that in $I(x) \cap Y_{j+2 r+2}$ there is an odd number of points to the left of the line $X_{4 t}$ and an even number to the right (or the other way around). However, for the two points in $Y_{k} \cap X_{4 t-1}$ and $Y_{k} \cap X_{4 t+1}$ the identifying sets are mirror images with respect to the line $X_{4 t}$, and therefore cannot be the same.

Remark. The only values of $r$ for which better constructions are known are $r=3$ and $r=5 ;$ see $[4]$.

\section{A general construction for the brick wall grid}

We first give a construction for $r$ a multiple of 4 , then adapt it to the cases $r \equiv 1,2$ or 3 $\bmod 4$.

Theorem 7 If $r \geq 8$ is divisible by four, then there is an $r$-identifying code with density $\frac{8 r-8}{9 r^{2}-16 r}$ in the brick wall (hexagonal) grid.

Proof. In this proof it is convenient to use the hexagonal representation of the grid. 
Let the lines $A_{i}, Y_{i}, Z_{i}$ be as in Figure 13, and let $X_{i}$ always denote the vertical line that goes through the point in $A_{i} \cap Z_{i}$. We use the lines $X_{i}$ and $Y_{j}$ to indicate the locations of the vertices: we denote by $(i, j)$ the unique vertex in the intersection of $X_{i}$ and $Y_{j}$.

Denote

$$
L(0,0)=\{(0,0),(2,0), \ldots,(2 r-4,0)\}
$$

and in general

$$
L(i, j)=(i(2 r-4+r / 4), i r / 2+j r)+L(0,0) .
$$

We show that $C$, the union of all $L(i, j), i, j \in \mathbb{Z}$, is an $r$-identifying code.

The dimensions of the tile become $r / 2 \times(2 r-4+r / 4)$. Each tile contains $r-1$ codewords, which gives the claimed density.

We denote the union of the lines $Y_{0}, Y_{1}, \ldots, Y_{r}$ by $Y$.

One readily checks that for the vertex $y \in Y_{0} \cap Z_{k}$ the intersection $B_{r}(y) \cap Y$ is the finite area restricted by the lines $Y_{0}, Y_{r}, A_{k+r}$ and $Z_{k-r}$, and that for the vertex $z \in Y_{r} \cap Z_{k}$ the intersection $B_{r}(z) \cap Y$ is the finite area restricted by the lines $Y_{0}, Y_{r}, A_{k}$ and $Z_{k+r}$.

Consequently, the set of vertices in $Y$ that are covered by both the codewords $(2 r-$ $4,0) \in L(0,0) \cap Z_{2 r-4}$ and $(2 r-4, r) \in L(0,1) \cap Z_{3 r / 2-4}$ consists of the area restricted by $Y_{r}, A_{3 r-4}, Z_{5 r / 2-4}, Y_{0}, A_{3 r / 2-4}$ and $Z_{r-4}$. Moreover, one easily verifies that all these points are within distance $r$ from the left-most codeword $(2 r-4+r / 4, r / 2)$ of $L(1,0)$ and the vertex $(2 r-4-r / 4, r / 2)$.

Assume that $x$ is the unknown vertex, and that we know $I(x)$. We now show that based on $I(x)$ we can unambiguously identify $x$.

In particular, for every vertex $x$, we know there are indices $i$ and $j$ such that $I(x)$ intersects both $L(i, j)$ and $L(i, j+1)$. Without loss of generality, assume that $i=j=0$. In particular, we then have $x \in Y$.

Case 1: Assume first that $I(x)$ contains neither $(0,0)$ nor $(2 r-4,0)$. Then $x \notin Y_{0}$. The case when $I(x)$ contains neither $(0, r)$ nor $(2 r-4, r)$ is symmetric. We know that the set $I(x) \cap Y_{0}$ is symmetric with respect to the vertical line passing through $x$; hence we can immediately deduce on which vertical line $X_{h}$ the point $x$ is. Without loss of generality, let us assume that $X_{h}$ passes through $(r-2,0)$ (the middle point of $L(0,0)$ ) or is to the right of it. If we look at any point $y \in X_{h} \cap A_{2 k}, h<2 k<h+r / 2$, we know by the construction that the point in $Y_{0} \cap A_{2 k-r}$ is a codeword, and $x$ is the point in $X_{h} \cap A_{2 k}$ or below it if and only if $I(x)$ contains the codeword in $Y_{0} \cap A_{2 k-r}$. Similarly, $x$ is the point in $X_{h} \cap A_{2 k}, h<2 k<h+r / 2$, or above it if and only if $I(x)$ contains a certain codeword in $L(0,1)$. If $x \in Y_{r}$, we immediately know it, because then $x$ is covered by codewords from $L(0, s)$ for three different values of $s$. It is now clear that in all cases we can identify $x$.

Case 2: Assume second that $I(x)$ contains both $(2 r-4,0)$ and $(0, r)$. The case when $I(x)$ contains both $(2 r-4, r)$ and $(0,0)$ is symmetric. Since $x$ is within distance $r$ from $(2 r-4,0)$, it has to lie on the line $Z_{r-4}$ or to the right of it. Since $x$ is within distance $r$ from $(0, r)$, it has to lie on the line $Z_{r / 2}$ or to the left of it. Because $r \geq 8$, we have $r-4>r / 2$, unless $r=8$. If $r=8, x \in Z_{4}$, and the case is clear from Figure 13 .

Case 3: By Case 1, we can assume that $I(x)$ contains at least one of the points $(0,0)$ and $(2 r-4,0)$ and at least one of the points $(0, r)$ and $(2 r-4, r)$. By Case 2 , we can 
assume without loss of generality that $(2 r-4,0) \in I(x),(2 r-4, r) \in I(x),(0,0) \notin I(x)$, and $(0, r) \notin I(x)$. By taking the right-most point of $L(0,1)$ that does not cover $x$, we find an index $k$ such that $x \in Z_{2 k} \cup Z_{2 k-1}$. By taking the right-most point of $L(0,0)$ that does not cover $x$, we find an index $h$ such that $x \in A_{2 h} \cup A_{2 h-1}$. The hexagonal grid is a bipartite graph, and $A_{2 h} \cap Z_{2 k-1}$ is empty, likewise $A_{2 h-1} \cap Z_{2 k}$. So we know that $x$ is either the point in $A_{2 h} \cap Z_{2 k}$ or the point in $A_{2 h-1} \cap Z_{2 k-1}$. In particular, we already know, whether or not $x$ lies on a line $Y_{j}$ for some $j \geq r / 2$. Without loss of generality, assume that it does not. We know that $x \in A_{2 h-1} \cup A_{2 h}$. We have already seen that the fact that $(2 r-4,0)$ and $(2 r-4, r)$ both belong to $I(x)$ implies that $x$ is within distance $r$ from both $(2 r-4+r / 4, r / 2)$ and $(2 r-4-r / 4, r / 2)$. Because $r \geq 8$, the distance from the point $(2 r-4-r / 4, r / 2)$ to the right-most point of $L(1,0)$ is $r / 2+2 r-4 \geq 2 r$, and, moreover, the only point within distance $r$ from both $(2 r-4-r / 4, r / 2)$ and the right-most point of $L(1,0)$ is one of the codewords in $L(1,0)$, which we can immediately identify. Hence we can assume that $x$ is not covered by the right-most point of $L(1,0)$. This means that we can take the left-most point of $L(1,0)$ that does not cover $x$, and obtain an index $t$ such that $x \in A_{2 t} \cup A_{2 t+1}$. But the intersection of $A_{2 t} \cup A_{2 t+1}$ and $A_{2 h} \cup A_{2 h-1}$ gives us the unique index $i$ for which $x \in A_{i}$. Then we of course know, whether $x$ is the point in $A_{2 h} \cap Z_{2 k}$ or the one in $A_{2 h-1} \cap Z_{2 k-1}$.

Theorem 8 In the hexagonal grid, for $r \geq 9$, there is an $r$-identifying code with density

$$
\begin{gathered}
\frac{8}{9 r-25} \quad \text { if } r \equiv 1 \quad \bmod 4 ; \quad \frac{8}{9 r-34} \quad \text { if } r \equiv 2 \bmod 4 ; \\
\frac{8 r-16}{(r-3)(9 r-43)} \quad \text { if } r \equiv 3 \quad \bmod 4 .
\end{gathered}
$$

Sketch of the proof. Let $s$ be a multiple of 4 . Consider the code $C$ defined in the previous proof, and define

$$
\begin{gathered}
L^{\prime}(0,0)=L(0,0) \cup\{(2 s-2,0)\}, \quad L^{\prime \prime}(0,0)=L^{\prime}(0,0) \cup\{(2 s, 0)\}, \\
C^{\prime}=\bigcup_{i, j}\left(L^{\prime}(0,0)+(i(2 s-4+s / 4), i s / 2+j s)\right), \\
C^{\prime \prime}=\bigcup_{i, j}\left(L^{\prime \prime}(0,0)+(i(2 s-4+s / 4), i s / 2+j s)\right) .
\end{gathered}
$$

In other words, we add one or two codeword(s) to the right of each subcode $L(i, j)$ and keep the same tile and translation.

We claim that $C^{\prime}$ is $(s+1)$ - and $(s+2)$-identifying, and that $C^{\prime \prime}$ is $(s+3)$-identifying. The density of $C^{\prime}$ is $8 s /\left(9 s^{2}-16 s\right)$, which is equal to $8 /(9 r-25)$ when $r=s+1$ and to $8 /(9 r-34)$ when $r=s+2$. The density of $C^{\prime \prime}$ is $8(s+1) /\left(9 s^{2}-16 s\right)$, equal to $(8 r-16) /(r-3)(9 r-43)$ for $r=s+3$.

The proof of the fact that $C^{\prime}$ (respectively, $\left.C^{\prime \prime}\right)$ is $(s+1)$ - and $(s+2)$-identifying (respectively, $(s+3)$-identifying) is very similar to the previous proof and presents no 
further interest; therefore, it is omitted here. We simply draw the reader's attention to the following: the proof can be divided into the same three cases as in the proof of Theorem 7 , by considering the left-most and right-most codewords of $L(0,0)$ and $L(0,1)$ (here and in the following, $L$ is used - improperly - to designate $L^{\prime}$ or $L^{\prime \prime}$ ). Since the radius is increased by 1,2 or 3, we must add to the set $Y$ the lines $Y_{-1}$ and $Y_{s+1}$, then $Y_{-2}$ and $Y_{s+2}$, then $Y_{-3}$ and $Y_{s+3}$, in order to be able to conclude that $x \in Y$ if $I(x)$ intersects both $L(0,0)$ and $L(0,1)$.

In Case 1, we cannot immediately know when $x \in Y_{s}$, because other vertices are also covered by both $L(0,0), L(0,1)$ and $L(0,2)$. However the argument involving the lines $A_{i}$ suffices, plus in some cases the following argument: within a small triangle located at the upper part of $Y$, in the middle of $L(0,1)$, two points spotted by $L(0,0)$ can both be covered by all points in $L(0,1)$ and therefore are not immediately separated, but then $L(0,2)$ separates them. The point of adding codeword(s) to the right of $L(0,1)$ is precisely to reduce the size of this triangle so that $L(0,2)$ can fully work on it.

In Case 2, the subscripts for the lines $Z$ change a little but remain in $s$ and $s / 2$, and the small cases (where the spheres centred at the left-most codeword of $L(0,1)$ and at the right-most codeword of $L(0,0)$ intersect) can be checked by hand.

Case 3 is very similar and we get pairs of points which, except for small values of the radius, will be separated by $L(1,0)$. For these small values, other subcodes $L(i, j)$ intervene.

Remark. For small values of $r$ (up to 30), there exist specific, better constructions, see $[4]$.

\section{Conclusion}

Gathering the general lower (Theorems 1-3) and upper (Theorems 4-8) bounds obtained here, plus the upper bound established in [13] in the case of the square lattice, we obtain the following results. For the brick wall grid:

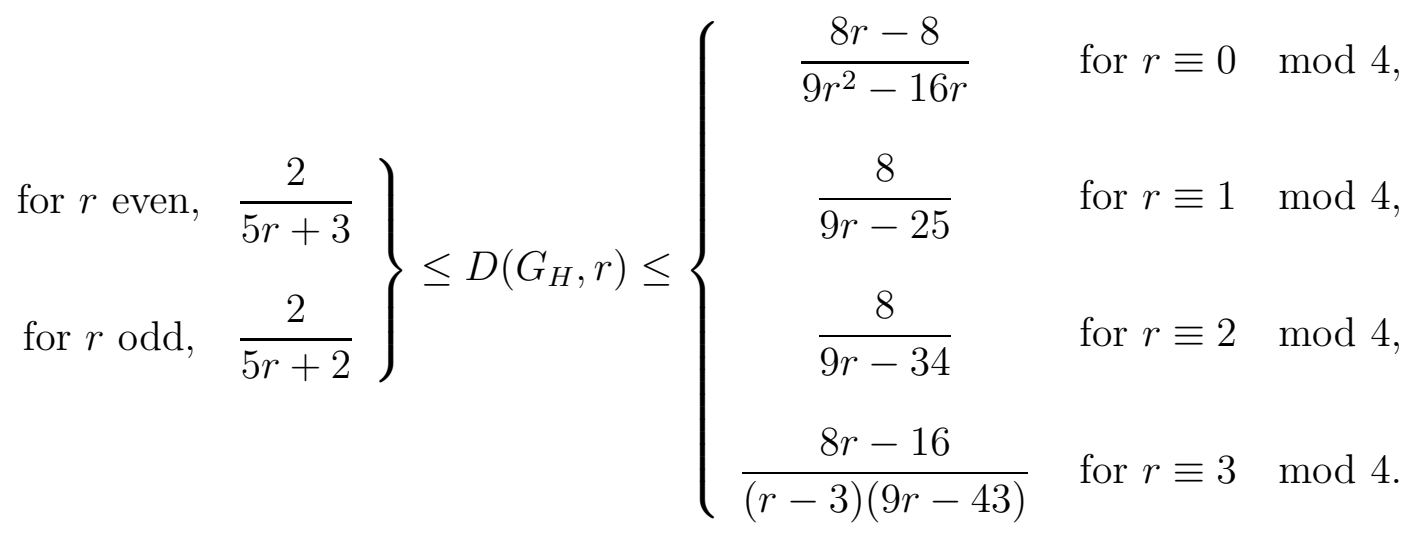

For the square lattice: 


$$
\frac{3}{8 r+4} \leq D\left(G_{S}, r\right) \leq\left\{\begin{array}{cl}
\frac{2}{5 r} & \text { for } r \text { even } \\
\frac{2 r}{5 r^{2}-2 r+1} & \text { for } r \text { odd }
\end{array}\right.
$$

For the triangular lattice:

$$
\frac{2}{6 r+3} \leq D\left(G_{T}, r\right) \leq\left\{\begin{array}{lc}
\frac{1}{2 r+4} & \text { for } r \equiv 0 \bmod 4 \\
\frac{1}{2 r+2} & \text { for } r \equiv 1,2 \text { or } 3 \quad \bmod 4
\end{array}\right.
$$

When $r$ goes to infinity, we see that

$$
\begin{aligned}
2 / 5 r & \lesssim D\left(G_{H}, r\right) \lesssim 8 / 9 r \\
3 / 8 r & \lesssim D\left(G_{S}, r\right) \lesssim 2 / 5 r \\
1 / 3 r & \lesssim D\left(G_{T}, r\right) \leq 1 / 2 r .
\end{aligned}
$$

For small values of $r$, see [4].

\section{References}

[1] U. Blass, I. Honkala, S. Litsyn: On binary codes for identification, Journal of Combinatorial Designs, vol. 8, pp. 151-156, 2000.

[2] U. Blass, I. Honkala, S. Litsyn: Bounds on identifying codes, Discrete Mathematics, to appear.

[3] I. Charon, I. Honkala, O. Hudry, A. Lobstein: The minimum density of an identifying code in the king lattice, Discrete Mathematics, submitted.

[4] I. Charon, O. Hudry, A. Lobstein: Identifying codes with small radius in some infinite regular graphs, submitted.

[5] G. Cohen, S. Gravier, I. Honkala, A. Lobstein, M. Mollard, C. Payan, G. Zémor: Improved identifying codes for the grid, Electronic Journal of Combinatorics, Comments to 6(1), R19, 1999.

[6] G. Cohen, I. Honkala, A. Lobstein, G. Zémor: New bounds for codes identifying vertices in graphs, Electronic Journal of Combinatorics, vol. 6(1), R19, 1999.

[7] G. Cohen, I. Honkala, A. Lobstein, G. Zémor: Bounds for codes identifying vertices in the hexagonal grid, SIAM Journal on Discrete Mathematics, vol. 13, pp. 492-504, 2000 . 
[8] G. Cohen, I. Honkala, A. Lobstein, G. Zémor: On codes identifying vertices in the two-dimensional square lattice with diagonals, IEEE Transactions on Computers, vol. 50, pp. 174-176, 2001.

[9] G. Cohen, I. Honkala, A. Lobstein, G. Zémor: On identifying codes. In: Proceedings of the DIMACS Workshop on Codes and Association Schemes, DIMACS Series in Discrete Mathematics and Theoretical Computer Science, vol. 56, AMS, Providence, pp. 97-109, 2001.

[10] G. Cohen, A. Lobstein, G. Zémor: Identification d'une station défaillante dans un contexte radio-mobile. In: Aspects Algorithmiques des Télécommunications (AlgoTel '99), Actes, pp. 19-22, 1999.

[11] G. Exoo: Computational results on identifying $t$-codes, Preprint, 1999.

[12] I. Honkala: On the identifying radius of codes. In: Proceedings of the 7th Nordic Combinatorial Conference (eds. T. Harju and I. Honkala), Turku, 1999.

[13] I. Honkala, A. Lobstein: On the density of identifying codes in the square lattice, Journal of Combinatorial Theory (B), to appear.

[14] M. G. Karpovsky, K. Chakrabarty, L. B. Levitin: On a new class of codes for identifying vertices in graphs, IEEE Transactions on Information Theory, vol. 44, pp. 599-611, 1998. 